The Enemy of my Enemy is my Friend:

2 Immune-Mediated Facilitation Contributes to Fitness of Co-Infecting Helminths

$11 \quad{ }^{1}$ Center for Infectious Disease Dynamics and Department of Biology, The Pennsylvania State University, University Park, 16082 PA, USA

${ }^{3}$ Dipartimento di Elettronica, Informazione e Bioingegneria, Politecnico di Milano, 20133

\title{
Milano, Italy
}

22 Corresponding author:

23 Isabella M. Cattadori

24 Center for Infectious Disease Dynamics and

25 Department of Biology

26 128W Millennium Science Complex, The Pennsylvania State University

27 University Park, PA 16802 USA

28 Tel.: +1-814-865-9594

29 E-mail: imc3@psu.edu 


\section{Abstract}

31 Our conceptual understanding of immune-mediated interactions between parasites is

32 rooted in the theory of community ecology. One of the limitations of this approach is that

33 most of the theory and empirical evidence has focused on resource or immune-mediated

34 parasite competition and yet, there is ample evidence of positive interactions between

35 species that could be generated by immune-mediated facilitation. Here, we develop an

36 immuno-epidemiological framework and apply it to longitudinal infection data of two

37 gastrointestinal helminths that infect a population of free-living rabbits to investigate,

38 through model testing, the mechanisms of immune-mediated facilitation in dual

39 infections. Simulations show that weakened, species-specific IgA antibody responses

40 and unequal, albeit low, IgA cross-reactions explain higher parasite intensities in dual

41 compared to single infections, for both helminths. Simulations also show that rabbits with

42 dual infections shed more free-living stages that survive fort longer in the environment,

43 implying greater onward transmission than hosts with single infections. These findings

44 support the hypothesis that the two helminths interact through immune-mediated

45 facilitation which contributes to greater fitness and the long-term co-circulation of both

46 species in the host population.

49 Keywords: Intensity of infection, shedding, antibodies, cross-reaction, climate 


\section{Introduction}

51 Interference between parasite species, mediated via the host's immune response, is one

52 of the processes frequently proposed to explain interactions between different parasite

53 species, or genetically diverse strains, that co-infect the same host [1-6]. In these

54 instances, immunity is expected to preferentially target the more abundant or virulent

55 parasite and, by reducing its intensity, attenuate the competition on the less abundant

56 species. The two species can still co-exist, but their relative fitness depends on the net

57 outcome of these interactions.

59 An alternative scenario to competition is where immune-mediated interactions lead to

60 facilitation of either one or both of the co-infecting species [7] (figure 1). For example,

61 many parasites can suppress or divert the immune response in favour of their own

62 survival [8], and this action can benefit a second parasite species through a bystander

63 effect $[9,10]$. Similarly, because of the polarization and function of different branches of

64 the immune system, the response developed against one species can reduce or prevent

65 the reaction against a co-infecting species [11-14]. In these interactions, the net benefit

66 obtained from co-infection is asymmetrical (figure 1c). The facilitator parasite can control

67 the immune response, or become its target, and in so doing facilitates the second

68 parasite species that profits more than the facilitator, and consequently has greater

69 fitness than when it is the sole parasite in the host. Symmetrical immune-mediated

70 facilitation emerges when both parasite species benefit from their co-infections (figure

71 1b). This can occur through the reduction, suppression or evasion of the immune

72 response against each species, which gains both through greater vital rates and

73 abundance [15-17]. Immune tolerance could be considered an extreme example of

74 facilitation since the host immunity is engaged in repairing the injuries caused by the

75 parasites rather than controlling the infections [18]. Importantly, under these scenarios a 
76 parasite will have a selective advantage when it infects hosts already parasitized with

77 the other species, so long as the facilitation is not so strong as to destabilize the

78 interactions [19] or reduce host survival [20,21].

80 From an ecological perspective, the extent of immune-mediated parasite interaction is

81 the outcome of two fundamental components. First, the immune response against one

82 parasite species that is diverted to fight other parasites, e.g. the loss of specific

83 antibodies that cross-react with other infections, and second, the immune constraints

84 this parasite species experiences by the presence of other species, e.g. the effect of

85 cross-reacting antibodies from other infections (figure 1b,c). Therefore, a positive net

86 impact for each species, including any parasite manipulation of the immune reactions,

87 would be expected to facilitate co-infections both within the host and among the co-

88 circulating parasites at the host population level. Theory has shown that in the absence

89 of strong competition between two parasite species, a weak immune-mediated

90 symmetrical facilitation increases the virulence/abundance of both parasites, however,

91 under stronger facilitation parasite growth escapes regulation and the system becomes

92 destabilized [19].

93

94 To test for immune-mediated interactions leading to facilitation, we applied an immuno-

95 epidemiological model to 23 years of infection data and asked whether the interaction

96 between two helminths in dual infected hosts could be explained by immune facilitation,

97 and whether there were positive consequences for parasite fitness, or onwards

98 transmission, at the host population level. We used two helminth species,

99 Trichostrongylus retortaeformis and Graphidium strigosum, that inhabit different

100 gastrointestinal niches of the European rabbit (Oryctolagus cuniculus), the small

101 intestine and stomach respectively, implying that they were unlikely to exhibit direct 
102 competition. While both helminths cause chronic infections, T. retortaeformis, and less

103 so G. strigosum, shows evidence of regulation of abundance and fecundity through host

104 immunity [22-27]. Interestingly, field studies found higher intensities of the first and to a

105 lesser extent the second in rabbits with both helminths, when compared to hosts with

106 single infections [28,29], suggesting a possible positive interaction between the two

107 species.

109 We investigated two hypotheses within the processes of immune regulation. If

110 symmetrical facilitation is important, we should expect both helminth species from dual

111 infections to experience reduced immune constraints and higher abundance and/or

112 production of free-living stages, than parasites from single infections (figure $1 b$ ).

113 However, if asymmetrical facilitation drives the interaction between the two species, then

114 the dynamics for the facilitated parasite should be enhanced but the facilitator should not

115 show substantial changes, when compared to single infections (figure 1c). In the

116 opposite scenario of reduced dynamics, such as decreased intensity of infection and/or

117 parasite fitness for one of both species, this should support immune-mediated

118 interference.

120 We tested different candidate frameworks, and model selection was based on the ability

121 of each model to describe the observed dynamics of infection, while identifying a

122 parsimonious mechanism of host-parasite and parasite-parasite interactions. Since we

123 are interested in processes of facilitation through host immunity, rather than competition,

124 and given that the two helminths inhabit different gastrointestinal niches, parasite

125 interference for host resources, including manipulation of host's metabolism or the

126 interaction with the gut microbiome, were beyond the scope of this study. 
Material and Methods

130 Rabbits become chronically infected with the two helminths by eating herbage

131 contaminated with infective larvae; once in the host, larvae develop into adults that shed

132 eggs with the hosts' faeces (figure S1). Laboratory experiments showed that rabbits

133 develop a type 2 anti-inflammatory response against both species. However, while this

134 regulates both $T$. retortaeformis intensity and body growth, which then affects fecundity,

135 it appears to have a weaker effect against G. strigosum that maintains high intensities

136 throughout the infection [24-27]. A broader investigation of the immune profile in

137 experiments of infection and anthelminthic treatment found a down-regulation of genes

138 expressing type 1, type 2 and T-regulatory responses during reinfections with $G$.

139 strigosum and much less so with T. retortaeformis, both in single and dual infections

140 [23]. There was no evidence that G. strigosum contributed to this reduction, suggesting

141 that the host rather than the parasite was probably responsible for the observed immune

142 down-regulation [23]. The general conclusions that T. retortaeformis, and to a lesser

143 extent G. strigosum, is affected by host immunity were also proposed in field studies

$144 \quad[22,28-31]$.

146 The dynamics of the two helminths could also be negatively affected by ecological

147 processes of intra-specific competition for resources. Previous studies indicated that this

148 is probably not the main mechanism of regulation for $T$. retortaeformis but could play a

149 role for $G$. strigosum, especially at high abundances [26,31]. For example, rabbits

150 nutritionally constrained, through coprophagic restriction and on a fixed diet, carried the

151 same T. retortaeformis intensities as animals on a fixed diet only [32]. 
153 An important component of soil-transmitted helminths is the development and survival of

154 eggs and larvae in the environment. Temperature and rainfall affect the survival of both

155 T. retortaeformis and G. strigosum free-living stages [33-35] while at the host population

156 level, climate and seasonality were found to be important for subsequent infection and

157 thus parasite fitness [31].

\section{Model Datasets}

160 Here, we present the datasets and assumptions used for our mechanistic immuno161 epidemiological model, while in the next section we describe the model framework. The 162 study focused on a population of rabbits (population A) sampled monthly between 1980

163 and 2002 from our site in Scotland. For every rabbit collected, the abundance of $T$.

164 retortaeformis and G. strigosum was quantified by aliquots using standardized

165 parasitological techniques [36]. We classified rabbits with one helminth species as

166 single-infected and with both species as dual-infected. It is possible that some of the 167 adults with single infections may have been previously infected with the other species

168 that was subsequently eliminated. Rabbits do not appear to be able to clear $G$.

169 strigosum [23-25,28,37] but old hosts can remove T. retortaeformis [24,27], although in 170 endemic settings reinfection is relatively fast and the fraction of single infected hosts is 171 really quite low; nevertheless, dubious cases were removed. To avoid interference with 172 myxoma virus infection $[11,28]$ rabbits with myxomatosis symptoms were excluded. Host 173 age was arranged into eight classes, from age class 1 (one-month old) to age class 8

174 (8+ months old) $[22,28]$ and lifespan was considered to be about one year [38].

176 Population A provides 23 years of robust individual host data on the two helminths but 177 lacks information on host immunity. Since our aim is to integrate the immune response 178 of the rabbit with the dynamics of its parasites, we used data on serum antibody IgA and 
179 IgG data obtained from a second rabbit population (population B) located $\sim 5$ kilometres

180 away in a similar ecosystem and sampled for a much shorter time period (2004-2011),

181 following the same standardized procedures as in population A [29]. Antibody data were

182 available for six out of those eight years. Given its shorter time series of infection and

183 following a preliminary investigation (SI-1.3 and 1.4), antibodies from population B were

184 used to inform the parameterization of the immune response for the longer sampling of

185 population A. This was based on similar dynamics of the two helminths (compare figure

186 S2 with figure 2 and statistical results in SI-1.3 and 3.3) and the rabbits' age structure

$187(\mathrm{SI}-1.4)$ at the two populations.

189 We selected species-specific IgA antibodies to represent host immunity against each of

190 the two helminths. Naturally, this is an over-simplification of the immune response, and it

191 was determined by the following reasons. First, IgA is the most abundant

192 immunoglobulin at mucosal surfaces, including the lamina propria of the gastrointestinal

193 tract where it plays a critical role in the humoral response against gut infections [39].

194 Second, $\lg \mathrm{A}$ is an important contributor to the regulation of helminth abundance and vital

195 rates [40-45], where the degree of protection is strongly affected by the host-helminth

196 system and its history of infection [46-48]. In this system, we found that $\lg A$ (but not $\lg G$ )

197 follows the dynamics of the two helminths, suggesting a rapid response and a

198 reasonable representation of the immune response that does exhibit some regulatory

199 proprieties but no long-term protection [23-27]. Third and most important, we needed a

200 variable with an antigenic-specific immune response that could allow the quantification of

201 separate reactions, namely species-specific and cross-reacting signals and, as such,

202 would capture the essence of symmetric/asymmetric interactions, while avoiding

203 additional complexities of adding extra variables and unnecessary assumptions. Our

204 selection was also for a variable that could be easily quantified from large field datasets. 
205 Moreover, antibodies are tractable model tools since we can select among different

206 isotypes based on the parasite-specific relationship of interest.

208 Species-specific serum IgA was originally quantified using excretory/secretory products

209 of adult parasites, as a source of antigen, and the enzyme-linked immunosorbent assay

210 (ELISA) [29]. Tests of in vitro antibody performance and competitive abilities between

211 the two helminths indicated good selective abilities to discriminate between the two

212 helminths when they co-occur [29]. Despite these in vitro analyses, we did not exclude

213 the possibility of antibody cross-reactivity, especially at high intensities of infection.

214 Finally, weather variables on mean air temperature and relative humidity were collected

215 daily from the nearby Hutton Institute (UK).

218 We used a deterministic, age-structured, immuno-epidemiological model with climatic

219 forcing, previously selected among different candidate models for this rabbit-helminth

220 system $[30,31]$. The model was then expanded to analyse hosts with single and dual

221 infections and to explicitly include the contribution of species-specific and cross-reacting

222 IgA responses. The demography of the host population was also included. We assumed

223 that the within-host helminth dynamic can be captured as:

$225 \frac{\partial P_{i}(a, t)}{\partial t}+\frac{\partial P_{i}(a, t)}{\partial a}=\Phi(a) F_{i}(t) \exp \left[-\left(r_{i i} I_{i}(a)+r_{j i} I_{j}(a)\right)\right]\left(1-P_{i}(a, t) / \delta_{i}\right)^{+}-\left(\mu_{i}+\mu_{H}\right) P_{i}(a, t)$,

226 with $\left(1-P_{i}(a, t) / \delta_{i}\right)^{+}=\left\{\begin{array}{cc}\left(1-P_{i}(a, t) / \delta_{i}\right) & \text { if }\left(1-P_{i}(a, t) / \delta_{i}\right)>0 \\ 0 & \text { otherwise }\end{array}\right.$

228 where: the intensity of infection (IOI) of the focal helminth $P_{i}(i=T$ for $T$. retortaeformis or $229 i=G$ for $G$. strigosum), changes with host age, $a$ in days, and time, $t$. The intensity of the 
230 helminth-specific $\lg A$ response, $l_{i}$, changes with host age, $a ; \Phi(a)=\Phi_{0}\left(\frac{200+9 a}{3340}\right)^{\gamma}$ is an

231 allometric function describing the age-dependent host feeding rate, with $\Phi_{0}$ and $\gamma$ being

232 suitable constants for each helminth species [30]. Given the similar IgA responses to

233 infective third stage larvae (L3) and adults [24,25], and to avoid model redundancy, we

234 focused on adults, assuming that there is no time delay from the ingestion of $L 3$ to

235 parasite maturation. The $r$ parameters account for the effect of the immune response on

236 the parasite intensity of infection, specifically, $r_{i i} /(a)$ is the age-dependent and species-

237 specific $\lg A$ response that is stimulated by, and targets, parasite $i$, while $r_{j i}(a)$ represents

238 the age-dependent and species-specific $\lg A$ stimulated by parasite $j(j \neq i)$ that targets

239 parasite $i$ in dual-infected rabbits. $r_{i i}$ and $r_{j i}$ regulate the intensity of the stimulus triggered

240 by the parasite; setting $r_{j i}=0(j \neq i)$ accounts for single infections. IgA is modelled to impact

241 within-host parasite establishment and survival. Since the two helminths colonize

242 different niches we did not consider direct inter-specific competition although we

243 included the non-negative term $\left(1-P_{i}(a, t) / \delta_{i}\right)^{+}$, which quantifies the intra-specific

244 intensity-dependent effect on helminth establishment, with $\delta_{i}$ representing the carrying

245 capacity of helminth species $i . \mu_{i}$ is the species-specific natural mortality rate of

246 established parasites while $\mu_{H}$ is the host natural mortality rate $\left(\mu_{H}=0.0069\right.$ days $\left.^{-1}\right)$ [38];

247 the total mortality rate of parasites $\mu$ includes both $\mu_{i}$ and $\mu_{H}$.

$249 F_{i}(t)$ is the risk of infection $(\mathrm{RI})$ of the host by parasite $i$ and quantifies the density of free-

250 living helminths available for infection on the herbage at time $t . F_{i}(t)$ is driven by the

251 intensity of infection in the host, $P_{i}$, and the linear effects of air temperature, $\tau(t)$, and

252 relative humidity, $H(t)$, on the mortality of free-living stages, $\mu_{F i}(\tau(t), H(t))[31]$ as: 
$254 \frac{d F_{i}}{d t}=\alpha_{p i}^{\prime} \int_{a_{\min }}^{a_{\max }} e^{-\mu_{H} a} f(t-a) P_{i}(a, t) d a-\mu_{F i}(\tau(t), H(t)) F_{i}$

255

256 with $\alpha_{p i}^{\prime}=\alpha_{p i} s_{i} R$. The quantity $\alpha_{p i}^{\prime}$ describes the rate at which eggs are shed by an

257 infected host and includes the total annual recruitment of rabbits, $R$, into the host

258 population, the total number of eggs shed by an adult parasite per unit of time, $s$,

259 independent of host age, and the survival of free-living stages, $\alpha_{p i}$. In the model, eggs

260 hatch directly into infective stages to help reduce model complexity while retaining the

261 fundamental biological characteristics of the system and the emphasis on the within-host

262 processes, and yet permitting an estimate of parasite fitness. The relative impact of

263 weather on the loss of free-living stages is set as: $\mu_{F i}(\tau(t), H(t))=$

$264 \alpha_{0 i}-\alpha_{1 \tau i} \tau(t)-\alpha_{1 h i} H(t)$, where $\alpha_{0 i}$ represents the baseline natural parasite mortality rate,

265 while $\alpha_{1 \tau i}$ and $\alpha_{1 h i}$ depict changes in the mortality rates driven by temperature and

266 humidity, respectively [31]. The proportional change of $F_{i}(t)$ with the host intensity of

267 infection is described by the term $\int_{a_{\min }}^{a_{\max }} e^{-\mu_{H} a} f(t-a) P_{i}(a, t) d a$, where $a_{\max }=283$ days

268 is the maximum age of an individual recorded in the data and $a_{\min }=30$ days, which is

269 approximately the age when naïve rabbits switch from milk to herbage and are exposed

270 to infective stages. The first age class of naïve rabbits is initiated by assigning a null

271 intensity of both infection and $\lg A . F_{i}(t)$ explicitly depends on the age structure of the

272 host population and thus, accounts for host reproduction, $f(t)$, the relative number of

273 births at time $t$, and host survival, $e^{-\mu_{H} a}$, which represents the probability that rabbits are

274 still alive at age a. We modelled $f(t)$ as a beta probability density function calibrated

275 against the fraction of 2-months old rabbits (SI-2.1). We assumed that infections with the

276 two helminths occur through simultaneous ingestion, which is commonly expected in

277 natural conditions; eggs are also shed simultaneously [29]. Table 1 summarizes the

278 model variables and parameters. 
280 As the framework (Eqs. 1 and 2 ) is rather complex, and to identify the model that

281 represents the best compromise between complexity and data availability, we focused

282 on population A dataset to quantify the functional relationships and fundamental

283 mechanisms of helminth dynamics, and used population B for the details on the host

284 immune response. The first nontrivial problem we faced was fitting the model to data

285 from population $A$ with all the observed combinations of helminth abundances in dual-

286 infected rabbits, including changes in the proportion of hosts with single and dual

287 infections over time. To reduce this computational difficulty, the rabbit population was

288 grouped in four subsets of infection data and the model was independently fitted to

289 individual rabbits from each of these subsets: i)- rabbits with $T$. retortaeformis and free of

290 G. strigosum, ii)- rabbits with G. strigosum and free of T. retortaeformis; iii)- rabbits with

291 both parasites but fitting to $T$. retortaeformis data only and iv)- rabbits with both parasites

292 but fitting to G. strigosum data only. Animals free of both helminths at the time of

293 sampling, i.e. not currently infected, were included in every subset to provide the naïve

294 condition, once dubious cases were identified and removed.

296 A similar approach was used for assigning the IgA values to rabbits from population $A$.

297 The $\lg$ A data from population B were initially grouped into the four subsets and, for each

298 one, the continuous age-related immune values $I_{i}(a)$ and $l_{j}(a)$ were obtained by

299 interpolating a $4^{\text {th }}$ order polynomial function to the relationship between mean $\operatorname{lgA}$ and

300 host age, weighted by sample size (figure S2a and $b$ ). Different smoothing functions

301 were examined and the $4^{\text {th }}$ order polynomial fitted well our data. Rabbits from population

302 A were then assigned an interpolated $\lg \mathrm{A}$ value according to their type of infection and

303 age. 
305 The second non-trivial problem was related to model selection [49] and the possible

306 overfitting when using a model with many free parameters. We tested five hypothesis-

307 driven models (three for single infections) that represented different mechanisms of

308 parasite regulation: i)- no constraints and parasites are affected by birth-death

309 processes, ii)- immune-mediated constraints through IgA responses, iii)- intra-specific

310 intensity-dependent constraints for host's resources and, for the dual-infected hosts, we

311 also examined iv)- cross-immunity via IgA responses (table 2). The complexity of the

312 framework was explored by considering models that included these mechanisms in

313 different combinations. Model selection and parameter calibration were performed

314 independently for each of the four subsets using individual data from population A.

316 To increase model adherence to observed data the shape parameter $\gamma$, pertaining the

317 rabbit's feeding rate, $\Phi(a)$, was initially tuned by fitting the most complex model (M2 for

318 single and M4 for dual infections) to the annual mean intensities of infection by host age,

319 simultaneously in single and dual infections of each helminth species. We then used this

320 value for all the models, including the less complex ones. During model fitting, $\gamma$ was

321 kept fixed while all the other parameters were recalibrated to the individual data of each

322 of the four subsets. The best-performing model formulation was selected using the

323 Akaike Information Criterion [49] and the lowest AIC. To allow the system to reach

324 regime conditions during calibration, the model was simulated for an initial warm-up of

325 23-years that was then removed, full details of this procedure are reported elsewhere

326 [31].

328 The numerical integration of the model was achieved using MATLAB® ode45 solver

329 function based on an explicit Runge-Kutta solution with adaptive time step size [50]. For

330 each of the four subsets of data, we compared the empirical helminth intensity in every 
331 rabbit to the expected intensity of infection by time and age of every host provided by the

332 model output, assuming that the intensity of infection is distributed as a negative

333 binomial [28] with age- and time-dependent mean $P(a, t)$ and aggregation parameter $k$,

334 calibrated together with the model parameters [28]. The parameters combination that

335 maximized the likelihood function was selected using a non-linear solver based on the

336 Nelder-Mead simplex algorithm [51].

338 Finally, to provide a statistical measurement of the differences between single and dual

339 infected rabbits, the model simulated quantities of interest, namely, intensity of infection,

340 abundance of eggs shed, risk of infection (i.e. viable free-living stages) and antibody

341 response, were compared using Generalized Linear Models (GLM, with normal or

342 negative binomial distribution of errors). Type of infection (single or dual) was entered as

343 categorical variable, while host age was included as a continuous variable. The additive

344 effects and two-way interactions of the explanatory variables were examined. For

345 consistency, the same analysis was repeated using the empirical data (SI-1.2).

\section{Results}

348 Here we present simulations from the best selected model and parameter calibration on

349 population A, the focus of our study. All the statistical results are reported in SI-3.

\section{Epidemiology of Single and Dual infections}

352 The model that best described the four subsets of infection data comprised the species-

353 specific IgA response and the intra-specific intensity-dependence for single infections,

354 dual infections also included IgA cross-reaction between the two helminths (table 2, SI-

355 3.1). Individual-based simulations captured the average trends of infection by host age

356 (figure 2), but less so the large intra-annual variation observed in the empirical data 
357 (figure S6). For both helminths the simulated intensities by host age were significantly

358 higher in rabbits with dual than single infections (table S3). T. retortaeformis rapidly

359 accumulated with host age and maintains high intensities in older rabbits (figure 2a),

360 while accumulation of $G$. strigosum intensities was slower (figure $2 b$ ). Consistent with

361 these model results, the empirical intensities of infection by host age were significantly

362 higher in rabbits with dual than single infection, for both helminths (figure 2, table S3).

Immune response and Immune-mediated parasite facilitation

365 The estimation of immunological parameters yields information on the immunological

366 mechanism that could generate the epidemiological patterns observed. Simulations

367 indicate that the stimulus to develop a specific $\lg A$ response to $T$. retortaeformis is

368 stronger in rabbits with single than dual infections $\left(r_{T T}=0.734\right.$ vs 0.338 , table 3$)$. In this

369 latter group, the cross-reaction stimulus for a specific IgA response against G. strigosum

370 that also attacks $T$. retortaeformis is essentially null $\left(r_{G T}=0.00016\right)$. The projected

371 relationships between intensity of infection and IgA stimuli indicate that a proportional

372 increase in the specific IgA stimulus $\left(r_{T T}\right)$ will reduces $T$. retortaeformis intensities, while

373 the stimulus for an IgA response to G. strigosum that cross-reacts with $T$. retortaeformis

$374\left(r_{G T}\right)$ remains extremely weak and will have no impact on this helminth (figure $\left.3 a\right)$. When

375 we consider the estimated species-specific antibody response against $T$. retortaeformis,

$376 r_{T T} l$, values are significantly lower in dual than single infection, and for rabbits of all age

377 groups (figure $4 a$, table S4). These results suggest that the higher T. retortaeformis

378 intensities in dual-infected hosts are caused by the weakening of the stimulus to a

379 specific IgA response that is diverted against $G$. strigosum (also see below) and a

380 negligible immune-mediated interference by this latter helminth in the form of a weak

381 cross-reaction, $r_{G T} l$. We note that, while empirical data only quantify specific IgA's, the

382 model allows us to quantify both the species-specific $\left(r_{i i} l_{i}\right)$ and the cross-reactive $\left(r_{j i} l_{j}\right)$ 
383 relative strength of $\lg A$, namely the effect of an increase (or decrease) of $\lg A$ on the

384 intensity of infection.

386 The intra-specific carrying capacity, $\delta$, is higher in rabbits with dual than single infections

387 (789.74 vs 651.6, respectively, table 3) supporting the lower intensity-dependent control 388 in this latter group.

390 For G. strigosum, simulations showed that the stimulus to develop a specific $\lg A$

391 response is low (table 3$)$, and lower in rabbits with single $\left(r_{G G}=0.121\right)$ than dual infection

$392\left(r_{G G}=0.208\right)$. The stimulus for an $\lg A$ response specific to $T$. retortaeformis that cross-

393 reacts against $G$. strigosum is four times higher $\left(r_{T G}=0.804\right)$, implying interference from

394 this helminth. Moreover, the investigation of how G. strigosum intensities will change in

395 relation to changes in the antibody stimuli indicates that the simulated intensities will

396 decline with a proportional increase of $r_{T G}$ and less so of $r_{G G}$ (figure $3 b$ ). Examination of

397 the estimated antibody response, $r_{G G} l$, by host age showed that values are significantly

398 higher in rabbits with dual than single infections (figure $4 b$, table S4), although it is

399 important to observe that these values remain consistently low. All together, these

400 findings suggest that dual-infected rabbits should control G. strigosum more successfully

401 than single-infected hosts. However, the generally low IgA response, even for the cross-

402 reaction, could explain the significantly higher intensities observed in dual-infected hosts 403 (table S3).

404

405 Previous studies proposed that intensity-dependence could be more important for the 406 dynamics of this parasite [31]. Our simulations indicate that parasite carrying capacity is 407 slightly higher in hosts with dual than single infections ( $\delta=83.45$ and 78.71 , respectively, 
408 table 3), suggesting the tendency for weaker restrictions of G. strigosum intensities in

409 this latter group, as previously noted for T. retortaeformis.

411 Collectively, the weakening or fundamentally low $\lg \mathrm{A}$ responses, and the resulting higher

412 intensities of both helminths in dual infections, support the hypothesis of immune-

413 mediated facilitation between the two species. These higher intensities are generated

414 despite a clear uneven in the immune response against the two helminths.

417 Given the soil-transmitted nature of T. retortaeformis and G. strigosum, the density of

418 viable free-living stages on the pasture represents the risk of infection for hosts exposed

419 to these stages, and can be considered a proxy for onward transmission and parasite

420 fitness. The density of free-living stages is the sum of the abundance of eggs shed by

421 every host and their mortality rate. We used simulation results to examine whether

422 parasite immune-mediated facilitation has symmetrical or asymmetrical consequences

423 for parasite fitness. The estimated abundance of eggs shed on the pasture is

424 significantly higher in rabbits with dual than single infections, for both helminths (table

425 S5). Eggs shedding peaks in young and a decrease in older animals for T. retortaeformis

426 while there is a constant increase with host age for G. strigosum (figure S7a,b).

428 On the herbage, the population of free-living stages is affected by environmental factors

429 (biotic and abiotic) with higher mortalities for $T$. retortaeformis than G. strigosum (table

430 3). Throughout the study period, the mortality rate of free-living stages produced by

431 rabbits with single and dual infections is, respectively: $\mu_{F T}=3.52$ and 1.58 day $^{-1}$ for $T$.

432 retortaeformis and $\mu_{F G}=0.009$ and 0.003 days $^{-1}$ for $G$. strigosum. Part of this loss is 
433 driven by weather: temperature has a strong impact on $T$. retortaeformis $\left(\alpha_{1} \tau=0.445\right.$

434 and 0.207 for single and dual infections, respectively) while humidity is more relevant to

435 G. strigosum ( $\alpha_{1 \mathrm{H}}=0.004$ and 0.002). The ecological differences between the two

436 helminths are also clear from the baseline natural mortality rate of free-living stages, $\alpha_{0}$,

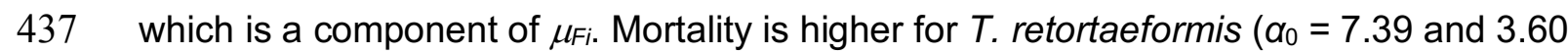

438 for single and dual infections, respectively) than for $G$. strigosum ( $\alpha_{0}=0.328$ and 0.194$)$,

439 indicating that larvae are on the pasture for a shorter period of time for the former

440 helminth. More important, simulations suggest that under analogous environmental

441 conditions, free-living stages from both helminths experience lower natural and climate-

442 driven mortality, and thus remain available for onward transmission for longer, when

443 derived from rabbits with dual infections (compare $\alpha_{0}$ and $\mu_{F i}$ from single and dual

444 infections).

446 There is strong seasonality in the accumulation of free-living stages on the herbage, and

447 hence the risk of infection (figure 5). T. retortaeformis accumulation is at the highest in

448 August primarily through the shedding of young and newly infected rabbits and drops in

449 June, coinciding with the peak of newly born rabbits (figure S3). G. strigosum shows the

450 highest densities around March, when the population is composed predominantly of

451 adults and the lowest around July, especially for dual infected hosts, with the arrival of

452 newborn hosts. In both cases, rabbits dual-infected generate significantly more viable

453 free-living stages throughout the year, than single-infected rabbits (table S6). Therefore,

454 while differences between the two helminths are expected because of their ecological

455 characteristics, both $T$. retortaeformis and G. strigosum from dual-infected rabbits exhibit

456 significantly higher fitness, including higher intensities of infection, than helminths from 
457 single infections, supporting the hypothesis of symmetrical immune-mediated facilitation

458 between the two parasites.

\section{Discussion}

461 We applied an immuno-epidemiological model to empirical data of two helminths from a

462 population of rabbits and the findings support the hypothesis that immune-mediated

463 facilitation can explain T. retortaeformis and G. strigosum higher intensities in rabbits

464 with dual compared to single infections. Dual infections are facilitated by weakened

465 species-specific IgA responses and unequal IgA cross reactions. Our multi-scale

466 approach also suggests a symmetrical immune-mediated facilitation where weak $\lg A$

467 stimuli contribute to greater number of eggs shed and higher survival of the free-living

468 stages, and thus higher transmission, when compared to parasites derived from hosts

469 with single infections. Given that rabbits with dual infections represent the large majority

470 of the sampled population (figure S4), the dynamics of the two helminths is primarily

471 driven by this group of hosts.

473 Cross-immunity, where protection to one species provides some defence against a

474 second species, has been proposed in a variety of systems [e.g. 1-3,6,52]. Our study

475 offers novel insights by showing that, although some immune-mediated interference

476 between two helminths is likely to occur, IgA cross-reacts disproportionately and has

477 small impact on parasite intensity, it is essentially null against $T$. retortaeformis and has

478 a low effect against G. strigosum. This pattern is consistent with theoretical work

479 showing that two parasites will increase abundance and persist in the host if immune-

480 mediated facilitation is not too strong to destabilize the system [19]. The evidence in

481 animal systems of positive interactions between parasite species [1,2,53], suggests 
482 some level of immune-mediated facilitation, irrespective of the specific immune

483 mechanism involved. Examples of facilitation through host immunity have been

484 frequently described for HIV associated co-infections in humans, such as HIV-malaria or

485 HIV-Tb $[58,59]$. Positive interactions between micro- and macro-parasites, mediated by

486 trade-offs in the immune functions and responses, are expected to benefit one or both

487 parasites [11,13]. For helminth co-infections, synergistic effects could emerge

488 throughout diverse processes; for example, the allocation of IgE against helminth

489 species could help explain the positive correlation between Ascaris lumbricoides and

490 both Trichuris trichiura and hookworm infections in humans (15, 54-56). Similarly, the co-

491 circulation of Trichostrongylus spp., Haemonchus contortus and Teladorsagia

492 circumcincta in domestic and wild animal populations is facilitated by their immuno-

493 modulatory properties [57] and could be complemented by asymmetric immune

494 reactions.

496 We can expect that one of the consequences of immune-mediated facilitation will be

497 greater parasite fitness. We found that rabbits with dual-infections contribute to a larger

498 number of free-living stages that are available for onward transmission, than did rabbits

499 with single infection. Our model did not explicitly quantify the relationships between $\lg A$

500 and parasite size or fecundity, where both fecundity and shedding are directly related to

501 parasite body length $[23,29]$, but coupled shedding to infection intensity, which is then

502 modulated by $\lg$ A. Some of these relationships were previously examined and showed a

503 negative relationship between $\lg A$ and $G$. strigosum body length in single infections and

504 between $\lg \mathrm{A}$ and $T$. retortaeformis body length or abundance in co-infections [23]. A

505 combination of positive and negative relationships was also found between the vital

506 rates of both helminths and type 1, type 2 and T-regulatory immune variables [23],

507 confirming the complex interactions between parasite demography and the host immune 
508 response. Indeed, the immune response to an infection is the results of a large number

509 of functions and factors, each of which has distinct roles and degree of specificity. Our

510 model framework describes a small constituent of this immune network, and gratifyingly

511 captured the effects, while other immune processes could also have potentially

512 contributed to the weaker net response of dual infected rabbits. More broadly, our results

513 are consistent with studies from other systems on the importance of IgA to helminth

514 growth, fecundity and shedding [60-63].

516 In addition to the diverse tolerance of the two helminths to weather [33-35], the higher

517 natural mortality of $T$. retortaeformis on the pasture is probably associated with the faster

518 life history of this parasite, namely, faster egg hatching rate [34] and faster within-host

519 maturation [64], when compared to G. strigosum [65]. Crucially, free-living stages

520 derived from rabbits with dual infections exhibited longer survival leading to higher

521 probability of onwards transmission. Laboratory studies showed that fewer antibodies

522 bind to eggs of $T$. retortaeformis than G. strigosum, and egg volume decreases for the

523 first and increases for the second during a single-dose infection experiment [66]. While

524 there was no clear evidence that antibodies altered egg size or hatchability, the impact

525 could be relevant in natural settings, when both the host and the helminths are under

526 environmental constraints. For instance, the low $\lg$ A specific response to $T$.

527 retortaeformis in hosts with dual-infections could lead to eggs of higher quality, such as

528 larger size or more tolerant to thermal changes, by females in better conditions. For $G$.

529 strigosum the mechanism is less clear but could also be related to improved female

530 conditions and enhanced egg quality with better tolerance to moisture variation.

532 Our data-informed modelling approach contributes to advances in the understanding of

533 immune-mediated facilitation on infection and transmission. The mechanistic 
534 understanding of these processes, and the contribution to parasite dynamics and fitness,

535 is still limited and in great need of empirical evidence. There is also a need to improve

536 the realism of modelling multiple infections, particularly for macro-parasites. The

537 proposed framework can be adapted to test alternative and more complex immune-

538 mediated formulations beyond the species examined in this study. T. retortaeformis and

539 G. strigosum share similarities with other gastrointestinal helminths of animals, including

540 human parasites, and our findings have relevance across a broad range of ecological

541 settings. The fundamental challenge is to identify the key variables that can clarify the

542 mechanisms of regulation across scales and influences parasite fitness. Gathering this

543 information can be daunting but is a prerequisite for laying the foundation of a better

544 understanding of the ecological role of co-infection in disease spread and persistence,

545 which is essential for developing control measures tailored on these groups of hosts.

\section{Acknowledgments}

548 This study was supported by the National Science Foundation (DEB-1145697). FD was

549 partially sponsored by The Ermenegildo Zegna Founder's Scholarship. We are grateful

550 to Tricia Brockman for creating figure S1.

\section{Ethic statement}

553 We used rabbit data already available from previous studies where sampling was performed

554 according to field procedures approved by the Institutional Animal Care and Use Committee of

555 The Pennsylvania State University (IACUC \# 26383 and 34489). All animal work adhered to the

556 guidelines laid out in the Guide for the Care and Use of Laboratory Animals. 8th ed. National

557 Research Council of the National Academies. National Academies Press Washington DC.

\section{References}

560 1. Cox, F.E.G. 2001. Concomitant infections, parasites and immune responses.

561 Parasitology 122, S23-S38.

562 2. Christensen, N.Ø., P. Nansen, B.O. Fagbemi and J. Monrad. 1987. Heterologous

563 antagonistic and synergistic interactions between helminths and between helminths 
564 and protozoans in concurrent experimental infection of mammalian hosts. Parasit.

565 Res. 73, 387-410.

566 3. Lee, T.D.G., R. K. Grencis and D. Wakelin. 1982. Specific cross-immunity between

$567 \quad$ Trichinella spiralis and Trichuris muris: immunization with heterologous infections and antigens and transfer of immunity with heterologous immune mesenteric lymph node cells. Parasitology 84, 381-389.

4. Gupta, S., J. Swinton, and R.M. Anderson. 1994. Theoretical studies of the effects of heterogeneity in the parasite population on the transmission dynamics of malaria. Proc. R. Soc. B. 256, 231-238.

5. Råberg, L., J.C. De Roode, A.S. Bell, P. Stamou, D. Gray, and A.F. Read. 2006. The role of immune-mediated apparent competition in genetically diverse malaria infections. Am. Nat. 168, 41-53.

6. Bhattacharyya, S., P.H. Gesteland, K. Korgenski, O.N. Bjørnstad, and F.R. Adler. 2015. Cross-immunity between strains explains the dynamical pattern of paramyxoviruses. PNAS 112, 13396-13400.

7. Zélé, F., S. Magalhães, S. Kéfi, and A.B. Duncan. 2018. Ecology and evolution of facilitation among symbionts. Nat. Com. 9, 4869.

8. McSorley, H.J. and Maizels, R.M., 2012. Helminth infections and host immune regulation. Clin. Microbiol. Rev. 25, 585-608.

9. Behnke, J.M., Wahid, F.N., Grencis, R.K., Else, K.J., Ben-Smith, A.W. and Goyal, P.K., 1993. Immunological relationships during primary infection with Heligmosomoides polygyrus (Nematospiroides dubius): downregulation of specific cytokine secretion (IL-9 and IL-10) correlates with poor mastocytosis and chronic survival of adult worms. Parasi. Immunol. 15, 415-421.

10. Genta, R.M. and Walzer, P.D., 1989. Strongyloidiasis. In Parasitic Infections in the Compromised Host (ed. Walzer, P. D. \& Genta, R. M.), pp. 463-525. New York: Marcel Dekker.

11. Cattadori, I.M., R. Albert, and B. Boag. 2007. Variation in host susceptibility and infectiousness generated by co-infection: the myxoma-Trichostrongylus retortaeformis case in wild rabbits. J.R.S. Interface 4, 831-840.

5965809. 
13. Graham A. 2008. Ecological rules governing helminth-microparasite coinfection. PNAS 105, 566-570.

14. Onah D.N. and Wakelin D., 1999. Trypanosome-induced suppression of responses to Trichinella spiralis in vaccinated mice. Int. J. Parasit. 29, 1017-1026.

15. Fleming, F.M., S. Brooker, S.M. Geiger, I.R. Caldas, R. Correa-Oliveira, P.J. Hotez, and J.M. Bethony. 2006. Synergistic associations between hookworm and other helminth species in a rural community in Brazil. Trop. Med. Intern. Health 11, 56-64.

16. Diedrich C.R. and J.L. Flynn 2011. HIV-1/mycobacterium tuberculosis coinfection immunology: how does HIV-1 exacerbate tuberculosis? Infect. Immun. 79, 14071417.

17. Shi, K., Li, H., Guo, X., Ge, X., Jia, H., Zheng, S. and Yang, H., 2008. Changes in peripheral blood leukocyte subpopulations in piglets co-infected experimentally with porcine reproductive and respiratory syndrome virus and porcine circovirus type 2. Vet. Microbiol. 129, 367-377.

18. Soares M.P., Teixeira L. and Moita L.F., 2017. Disease tolerance and immunity in host protection against infection. Nat. Rev. Immunol. 17, 83-96.

19. Eswarappa, S.M., S. Estrela and S.P. Brown. 2012. Within-host dynamics of multispecies infections: facilitation, competition and virulence. PLoS One 7: 38730.

20. Kamiya, T., N. Mideo, and S. Alizon. 2018. Coevolution of virulence and immunosuppression in multiple infections. J. Evol. Biol. 31, 995-1005.

21. Tillmann, H.L., Heiken, H., Knapik-Botor, A., Heringlake, S., Ockenga, J., Wilber, J.C., Goergen, B., Detmer, J., McMorrow, M., Stoll, M. and Schmidt, R.E., 2001. Infection with $\mathrm{GB}$ virus $\mathrm{C}$ and reduced mortality among HIV-infected patients. New Engl. J. Med., 345, 715-724.

22. Cattadori, I.M., B. Boag, O.N. Bjørnstad, S. Cornell, and P.J. Hudson. 2005. Peak

627 24. Murphy, L., N. Nalpas, M. Stear, and I.M. Cattadori. 2011. Explaining patterns of 628 infection in free living populations using laboratory immune experiments. Paras. 629 Immunol. 33, 287-302. 
25. Murphy, L., A.K. Pathak, and I.M. Cattadori. 2013. A co-infection with two gastrointestinal nematodes alters host immune responses and only partially parasite dynamics. Paras.Immunol. 35, 421-432.

26. Vanalli C., L. Mari, R. Righetto, R. Casagrandi, M. Gatto, and I.M. Cattadori. 2020 Within-host mechanisms of immune regulation explain the contrasting dynamics of two helminth species in both single and dual infections. Plos Comp. Biol. 16, p.e1008438.

27. Thakar, J., A.K. Pathak, L. Murphy, R. Albert, and I.M. Cattadori. 2012. Network model of immune responses reveals key effectors to single and co-infection dynamics by a respiratory bacterium and a gastrointestinal helminth. PLoS Comp. Biol. 8, p.e1002345.

28. Cattadori, I.M., B. Boag, and P.J. Hudson. 2008. Parasite coinfection and interaction as drivers of host heterogeneity. Int. J. Parasit. 38, 371-380.

29. Cattadori, I.M., B.R. Wagner, L.A. Wodzinski, A.K. Pathak, A. Poole, and B. Boag. 2014. Infections do not predict shedding in co-infections with two helminths from a natural system. Ecology 95, 1684-1692.

30. Cornell, S.J., O.N. Bjornstad, I.M. Cattadori, B. Boag, and P.J. Hudson. 2008. Seasonality, cohort-dependence and the development of immunity in a natural hostnematode system. Proc. R. Soc. B 275, 511-518.

31. Mignatti, A., B. Boag, and I.M. Cattadori. 2016. Host immunity shapes the impact of climate changes on the dynamics of two parasite infections. PNAS 113, 2970-2975.

32. Cattadori, I.M., A. Sebastian, H. Hao, K. Katani, I. Albert, K.E. Eilertson, V. Kapur, A. Pathak and S. Mitchell. 2016. Impact of helminth infections and nutritional constraints on the small intestine microbiota. PLoS One 11, 0159770.

33. Boag, B., and R.J. Thomas. 1970. The development and survival of free-living stages of Trichostrongylus colubriformis and Ostertagia circumcincta on pasture. Res. Vet. Sci. 11, 380-381.

34. Hernandez, A.D., A. Poole, and I.M. Cattadori. 2013. Climate changes influence freeliving stages of soil-transmitted parasites of European rabbits. Glob. Change Biol. 19, 1028-1042.

35. Gupta, S.P. 1961. The effects of temperature on the survival and development of the free-living stages of Trichostrongylus retortaeformis Zeder (Nematoda). Can. J. Zool. 391, 47-53. 
36. Boag, B. 1985. The incidence of helminth parasites from the wild rabbit Oryctolagus cuniculus (L.) in Eastern Scotland. J. Helminth. 59, 61-69.

37. Pathak, A.K., C. Pelensky, B. Boag, and I.M. Cattadori. 2012. Immuno-epidemiology of chronic bacterial and helminth co-infections: observations from the field and evidence from the laboratory. Int. J. Parasit. 42, 647-655.

38. Smith, G.C., and R.C. Trout. 1994. Using Leslie matrices to determine wild rabbit population growth and the potential for control. J. Appl. Ecol. 31, 223-230.

39. Mestecky, J. and McGhee, J.R., 1987. Immunoglobulin A ( $\lg A)$ : molecular and cellular interactions involved in IgA biosynthesis and immune response. Adv. Immunol. 40, 153-245.

40. Sorobetea, D., M. Svensson-Frej, and R. Grencis. 2018. Immunity to gastrointestinal nematode infections. Muc. Immunol. 11, 304-315.

41. Ben-Smith, A., Wahid, F.N., Lammas, D.A. and Behnke, J.M., 1999. The relationship between circulating and intestinal Heligmosomoides polygyrus-specific IgG1 and IgA and resistance to primary infection. Paras. Immunol. 21, 383-396.

42. Van Knapen, F., J.H. Franchimont, A.R. Verdonk, J. Stumpf, and K. Undeutsch. 1982. Detection of specific immunoglobulins ( $\lg G, \lg M, \lg A, \lg E$ ) and total $\lg E$ levels in human trichinosis by means of the enzyme-linked immunosorbent assay (ELISA). Am. J. Trop. Med. Hyg. 31, 973-976.

43. Clerc, M., Devevey, G., Fenton, A. and Pedersen, A.B., 2018. Antibodies and coinfection drive variation in nematode burdens in wild mice. Int. J. Parasit, 48, 785792.

44. Escribano, C., Saravia, A., Costa, M., Castells, D., Ciappesoni, G., Riet-Correa, F. and Freire, T., 2019. Resistance to Haemonchus contortus in Corriedale sheep is associated to high parasite-specific IgA titer and a systemic Th2 immune response. Sci. Rep., 9, 1-10.

45. Inaba T, et al. 2003. Monoclonal IgA antibody-mediated expulsion of Trichinella from the intestine of mice. Parasitology 126, 591-598.

46. Esser-von Bieren, J., I. Mosconi, R. Guiet, A. Piersgilli, B. Volpe, F. Chen, W.C. Gause, A. Seitz, J.S. Verbeek, and N.L. Harris. 2013. Antibodies trap tissue migrating helminth larvae and prevent tissue damage by driving IL-4Ra-independent alternative differentiation of macrophages. PLoS Pathog. 9, e1003771.

47. McCoy, K.D., M. Stoel, R. Stettler, P. Merky, K. Fink, B.M. Senn, C. Schaer, J. Massacand, B. Odermatt, H.C. Oettgen, and R.M. Zinkernagel. 2008. Polyclonal and 
specific antibodies mediate protective immunity against enteric helminth infection. Cell Host Microb. 4, 362-373.

48. Roach, T.I.A., K.J. Else, D. Wakelin, D.J. McLaren, and R.K. Grencis. 1991. Trichuris muris: antigen recognition and transfer of immunity in mice by $\lg \mathrm{A}$ monoclonal antibodies. Paras. Immunol. 13, 1-12.

49. Burnham, K.P., and D.R. Anderson. 2002. Model selection and multimodel inference: a practical information-theoretic approach. Springer, New York.

50. Dormand, J.R. and P.J. Prince. 1980. A family of embedded Runge-Kutta formulae.

$$
\text { J. Comp. Appl. Math. 6, 19-26. }
$$

\section{Lagarias, J.C., J.A. Reeds, M.H. Wright, and P.E. Wright. 1998. Convergence} properties of the Nelder-Mead simplex method in low dimensions. SIAM J. Optim. 9, 112-147.

52. Johnson, P.T. and I.D. Buller. 2011. Parasite competition hidden by correlated coinfection: using surveys and experiments to understand parasite interactions. Ecology 92, 535-541.

53. Petney, T.N. and R.H. Andrews. 1998. Multiparasite communities in animals and humans: frequency, structure and pathogenic significance. Int. J. Parasit. 28, 377393.

54. Booth, M., and D.A.P. Bundy. 1992. Comparative prevalences of Ascaris lumbricoides, Trichuris trichiura and hookworm infections and the prospects for combined control. Parasitology 105, 151-157.

55. Geiger, S.M., N.D.E. Alexander, R.T. Fujiwara, S. Brooker, B. Cundill, D.J. Diemert, R. Correa-Oliveira, and J.M. Bethony. 2011. Necator americanus and helminth coinfections: further down-modulation of hookworm-specific type 1 immune responses. PLoS Negl. Trop. Dis. 5, p.e1280.

56. Lepper, H.C., J.M. Prada, E.L. Davis, S.A. Gunawardena, and T.D. Hollingsworth. 2018. Complex interactions in soil-transmitted helminth co-infections from a crosssectional study in Sri Lanka. Trans. R. Soc. Trop. Med. Hyg. 112, 397-404.

57. McNeilly, T.N., and A.J. Nisbet. 2014. Immune modulation by helminth parasites of ruminants: implications for vaccine development and host immune competence.

7301606. 
731 59. Kwan, C.K. and J.D. Ernst. 2011. HIV and tuberculosis: a deadly human syndemic.

732 Clin. Microb. Rev. 24, 351-376.

733 60. Stear, M.J., S.C. Bishop, M. Doligalska, J.L. Duncan, P.H. Holmes, J. Irvine, L.

734 McCririe, Q.A. McKellar, E. Sinski, and M.A.X. Murray. 1995. Regulation of egg

735 production, worm burden, worm length and worm fecundity by host responses in

736 sheep infected with Ostertagia circumcincta. Parasit. Immunol. 17, 643-652.

737 61. Strain, S., S. Bishop, N. Henderson, A. Kerr, Q. Mackellar, S. Mitchell, and M. Stear.

738 2002. The genetic control of IgA against Teladorsagia circumincta and its association

739 with parasite resistance in naturally infected sheep. Parasitology 124, 545-522.

740 62. Henderson, N.G., and M.C. Stear. 2006. Eosinophil and IgA responses in sheep

741 infected with Teladorsagia circumcincta. Vet. Immunol. Immunopath. 112, 62-66.

742 63. McRae, K.M., M.J. Stear, B. Good, and O.M. Keane. 2015. The host immune

743 response to gastrointestinal nematode infection in sheep. Paras. Immunol. 37, 605-

$744 \quad 613$.

745 64. Audebert, F., H. Hoste, and M.C. Durette-Desset. 2002. Life cycle of

746 Trichostrongylus retortaeformis in its natural host, the rabbit (Oryctolagus cuniculus).

747 J. Helminth. 76, 189-192.

748 65. Massoni, J., J. Cassone, M.C. Durette-Desset, and F. Audebert. 2011. Development

749 of Graphidium strigosum (Nematoda, Haemonchidae) in its natural host, the rabbit

750 (Oryctolagus cuniculus) and comparison with several Haemonchidae parasites of

751 ruminants. Parasit. Res. 109, 25-36.

752 66. Lambert, K., A.K. Pathak. and I.M. Cattadori. 2005. Does host immunity influence

753 hatchability of helminth eggs shed in the environment? J. Helminth. 89, 446-452. 
754 Table 1. Variable and parameter definitions, and their units for model equations 1 and 2.

755 The symbols $a$ and $t$ represent age and time in days.

756

\begin{tabular}{|c|c|c|}
\hline $\begin{array}{l}\text { Variablel } \\
\text { parameter }\end{array}$ & Definition & Unit \\
\hline$P(a, t)$ & Intensity of infection & parasites $*$ rabbit $^{-1}$ \\
\hline$F(t)$ & Risk of infection & $\begin{array}{l}\text { parasites } * \text { (grass } \\
\text { unit }^{-1}\end{array}$ \\
\hline$\Phi(a)$ & Age-dependent host feeding rate & $\begin{array}{l}\text { grass unit } *(\text { day } * \\
{\text { rabbit })^{-1}}^{\text {ras }}\end{array}$ \\
\hline I(a) & Age-dependent host immune response & - \\
\hline Y & Allometric exponent of the host feeding rate & - \\
\hline$\Phi_{0}$ & Host feeding rate for reference body mass of $3340 \mathrm{~g}$ & $\begin{array}{l}\text { grass unit } *(\text { day } * \\
{\text { rabbit })^{-1}}^{\text {rat }}\end{array}$ \\
\hline$\mu_{i}$ & Natural mortality rate of parasite $i$ within the host & days $^{-1}$ \\
\hline$\mu_{H}$ & Host natural mortality rate & days $^{-1}$ \\
\hline$\delta$ & Parasite carrying-capacity & $\left(\text { parasites } * \text { rabbit }^{-1}\right)^{-1}$ \\
\hline$\mu_{F}$ & Weather-dependent mortality rate of free-living parasites & days $^{-1}$ \\
\hline$\alpha_{0}$ & $\begin{array}{l}\text { Parasite baseline mortality rate in the environment } \\
\text { associated with worm features }\end{array}$ & days $^{-1}$ \\
\hline$\alpha_{1 \tau}$ & Effect of temperature on mortality rate of free-living parasites & days $^{-1} *{ }^{\circ} \mathrm{C}^{-1}$ \\
\hline$\alpha_{1 h}$ & Effect of humidity on mortality rate of free-living parasites & $\begin{array}{l}\text { days }^{-1} * \text { units of } \\
\text { relative humidity }\end{array}$ \\
\hline$T(t)$ & Mean daily air temperature from min and max records & ${ }^{\circ} \mathrm{C}$ \\
\hline$H(t)$ & $\begin{array}{l}\text { Daily relative air humidity from dry-web bulb } \\
\text { temperature, atmospheric pressure at } 101.3 \mathrm{kPa}\end{array}$ & $\%$ \\
\hline$\alpha_{p}^{\prime}$ & Parasite shedding rate ${ }^{*}$ host total recruitment & $\begin{array}{c}\text { rabbit } * \text { (grass unit } * \\
\text { days) })^{-1}\end{array}$ \\
\hline$r_{i i}$ & Intensity of immune response to parasite $i$ triggered by $i$ & - \\
\hline$r_{j i}$ & Intensity of immune response to parasite $i$ triggered by $j$ & - \\
\hline$k$ & Parasite aggregation from negative binomial distribution & - \\
\hline
\end{tabular}


758 Table 2. Tested hypotheses and related mechanisms for single and dual infections of both

759 helminths with the corresponding model complexity $p$, indicated by the number of

760 parameters to be calibrated for each data subset.

761

\begin{tabular}{|c|c|c|c|c|}
\hline Models & Single infection & Dual infection & $\begin{array}{l}\text { Parameter } \\
\text { constraints }\end{array}$ & $p$ \\
\hline M0 & Parasite Birth-Death only & Parasite Birth-Death only & $\begin{array}{c}r_{j i}=0 \text { for any } j \text { and } i ; \\
\delta_{i}=\infty\end{array}$ & 8 \\
\hline M1 & Birth-Death + Specific Imm. & Birth-Death + Specific Imm. & $r_{j i}=0$ for $j \neq i ; \delta_{i}=\infty$ & 9 \\
\hline M2 & $\begin{array}{l}\text { Birth-Death + Specific Imm. } \\
+ \text { Intensity Depend. }\end{array}$ & $\begin{array}{l}\text { Birth-Death + Specific Imm. } \\
\text { +Intensity Depend. }\end{array}$ & $r_{j i}=0$ for $j \neq i$ & 10 \\
\hline M3 & - & $\begin{array}{l}\text { Birth-Death + Specific Imm. + } \\
\text { Cross Imm. }\end{array}$ & $\delta_{i}=\infty$ & 10 \\
\hline M4 & - & $\begin{array}{l}\text { Birth-Death + Specific Imm. + } \\
\text { Cross Imm. + Intensity Depend. }\end{array}$ & - & 11 \\
\hline
\end{tabular}


763 Table 3. Parameter values estimated from the best-fitted model for $T$. retortaeformis (TR)

764 and G. strigosum (GS) in single- (SI) and dual- (DU) infected hosts. Parameter definitions

765 are reported in table 1. $F(0)$ refers to the initial condition of the warm-up period of model

766 simulations while $y$ is included as a constant value. $\mathrm{AIC}=$ Akaike Information Criterion

767 value, $\mathrm{LL}=$ log-likelihood, RMSD= Root Mean Square Deviation.

768

769

\begin{tabular}{|c|c|c|c|c|}
\hline Parameters & TR-SI & $T R-\mathrm{DU}$ & GS-SI & GS-DU \\
\hline V & 3.09 & 3.09 & 5.73 & 5.73 \\
\hline$\Phi_{0}$ & 1.70 & 0.4738 & 1.84 & 0.430 \\
\hline$\mu_{i}$ & 0.0040 & 0.00219 & 0.212 & 0.0161 \\
\hline$\delta$ & 651.6 & 789.74 & 78.71 & 83.45 \\
\hline$F(0)$ & 76.42 & 6.28 & 61.30 & 3.28 \\
\hline$\alpha_{0}$ & 7.39 & 3.60 & 0.328 & 0.194 \\
\hline$\alpha_{1 \tau}$ & 0.445 & 0.207 & 0.0022 & 0.00019 \\
\hline$\alpha_{1 h}$ & -0.0003 & 0.00254 & 0.00375 & 0.00236 \\
\hline$\alpha_{p}^{\prime}$ & 1.86 & 3.322 & 0.129 & 0.1323 \\
\hline$k$ & 0.220 & 0.244 & 0.146 & 0.423 \\
\hline$r_{i i}$ & 0.734 & 0.338 & 0.1206 & 0.208 \\
\hline$r_{j i}$ & - & 0.00016 & - & 0.804 \\
\hline Parameters & 10 & 11 & 10 & 11 \\
\hline AIC & 17,074 & 19,204 & 3,052 & 10,878 \\
\hline LL & 8,624 & 9,591 & 1,516 & 5,428 \\
\hline RMSD & 576 & 999 & 49.2 & 73.4 \\
\hline
\end{tabular}


a

Single Infection
immune
regulation

Immune response

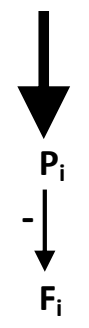

771

772 Figure 1. Scenarios of immune-parasite interaction in single infections (a) and dual-

773 infections ( $b$ and $c$ ) for two parasite species $\left(P_{i}, i=1,2\right)$ and consequences for their fitness

$774 \quad\left(F_{i}, i=1,2\right)$. Symmetrical and asymmetrical immune-mediated facilitation is presented for

775 the dual infection. We report: the magnitude (arrow thickness) and type of the effect (null=

7760 , sub-typical $=\sim$, positive $=+$, negative $=-$ ) and the parasite indirect interactions (dotted

777 arrow). a- Standard scenario were immunity directly affects parasite, $P_{i}$, traits (i.e.

778 abundance, development or fecundity) and reduces its fitness, $\mathrm{F}_{\mathrm{i}}$; examples: many

779 helminth species, including T. retortaeformis in rabbits (Cattadori et al. 2005). b- Immunity

780 decreases against each parasite, $P_{1}$ and $P_{2}$, and this benefits their fitness, $F_{1}$ and $F_{2}$,

781 compared to case $a ; \mathrm{P}_{1}-\mathrm{P}_{2}$ interactions (e.g. positive or unclear reaction) do not reduce

782 the positive net immune effect on $F_{1}$ and $F_{2}$; examples: hookworm and Ascaris

783 lumbricoides in humans (Fleming et al. 2006), HIV- Mycobacterium tuberculosis in

784 humans (Pawlowski et al. 2012), this study. c- Processes and impacts are as described

785 in case $b$, however, $\mathrm{P}_{1}-\mathrm{P}_{2}$ asymmetrical interactions benefit the fitness $\mathrm{F}_{2}$ of $\mathrm{P}_{2}$ but lead to

786 sub-typical ( ) or unclear $(0)$ increase in fitness $F_{1}$ for $P_{1}$, compared to case a; example:

787 Trichuris muris-Schistosoma mansoni in mice (Bickle et al. 2008) or $H$. polygyrus-

788 Trichinella spiralis in mice (Behnke et al. 1993). 

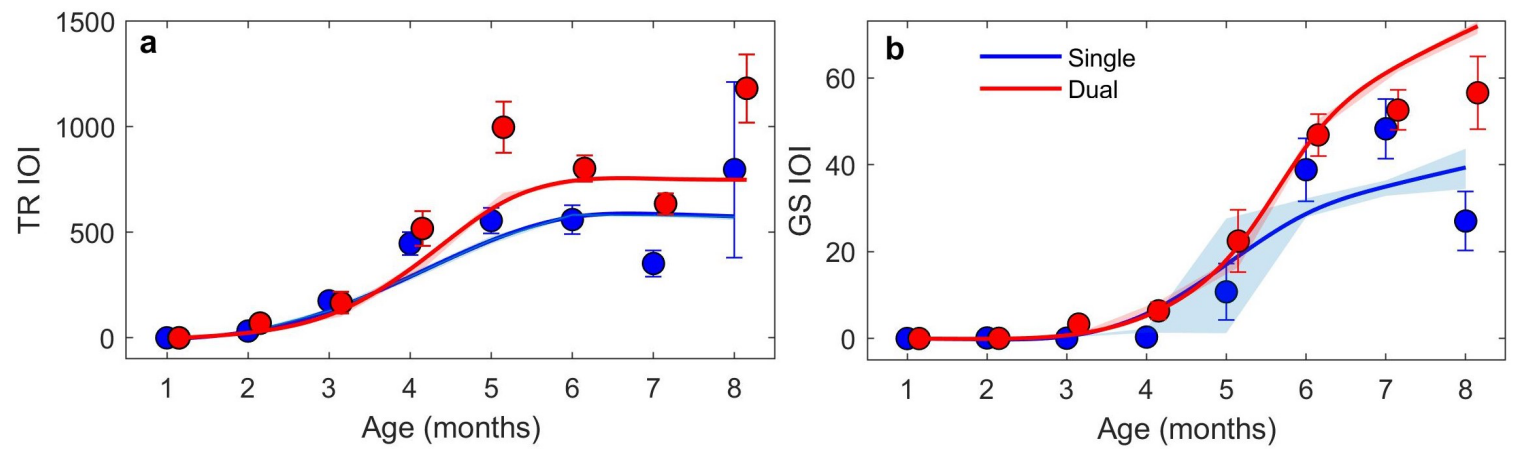

791

792 Figure 2. Relationship between intensity of infection (IOI) and host age for $T$. 793 retortaeformis (TR, a) and G. strigosum (GS, b) in single- (blue) and dual- (red) infected

794 hosts from population A. Mean and S.E. data are reported from individual-based model

795 simulations (lines and shadow bands) and field monitoring (circles and bars, these latter

796 ones calculated under the assumption that the data follow a negative binomial

797 distribution). Only simulations referring to collected rabbit data are reported. Small S.E.

798 bars and bands could be masked by circles or lines. IOI at age 1 is forced to start at 0

799 since rabbits are exposed to the risk of infection at about 30 days of age when they switch

800 from milk to herbage. 

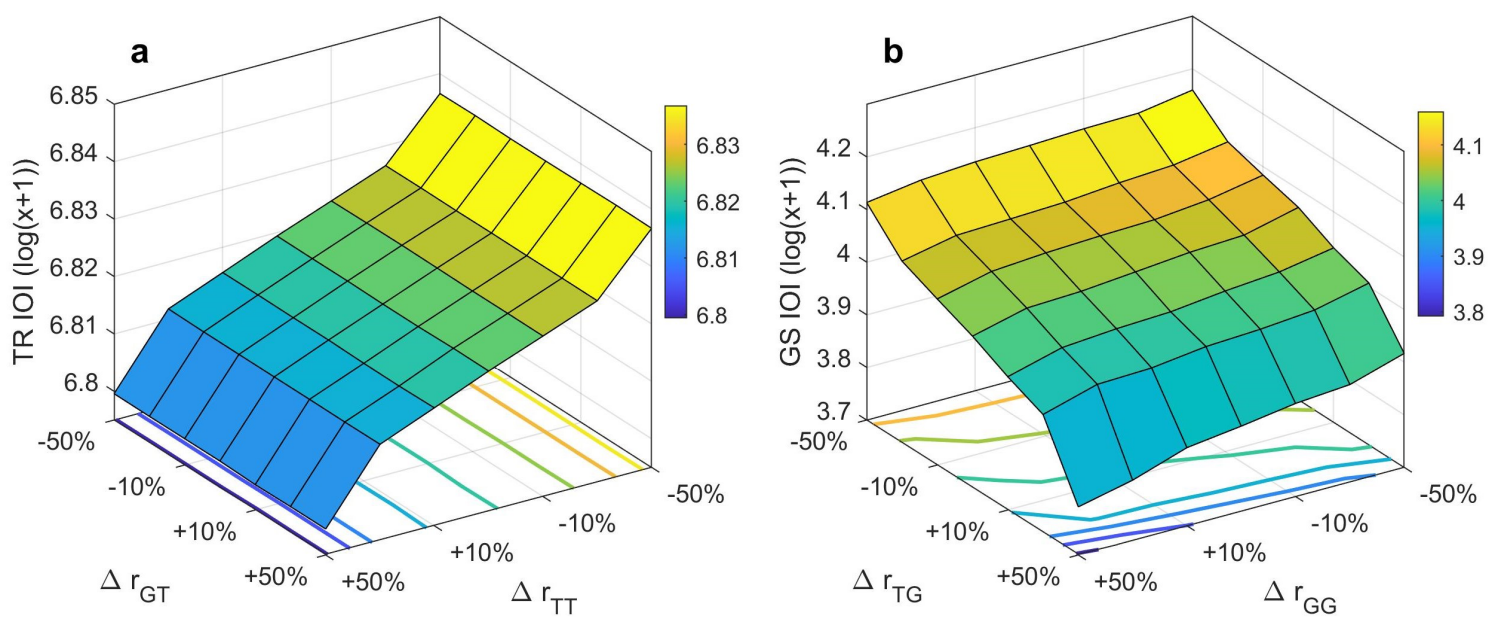

804

805 Figure 3. Three-way relationships among model-predicted intensity of infection (IOI) and

806 relative changes in species-specific $\left(\Delta r_{T T}\right.$ or $\left.\Delta r_{G G}\right)$ and cross-reacting $\left(\Delta r_{T G}\right.$ or $\left.\Delta r_{G T}\right)$ stimuli

807 to $\lg$ A production for T. retortaeformis (TR, a) and G. strigosum (GS, b). Predictions are

808 from the selected best model for dual infections (M4) fitted on population A. The bivariate

809 incremental variation (\% of increase or decrease) in the immune parameters, $r_{i i}$ or $r_{j i}$, is

810 relative to the baseline estimated values reported in table 3 . The IOls (heat surface) are

811 obtained via sensitivity analysis where the immune parameters are incrementally changed

812 one at a time, with respect to their estimated values, and simulations are run while holding

813 all the other parameters constant. The contour lines represent the predicted IOI values

814 from the heat map at specific $r_{i i}$ and $r_{j i}$ values. 
815
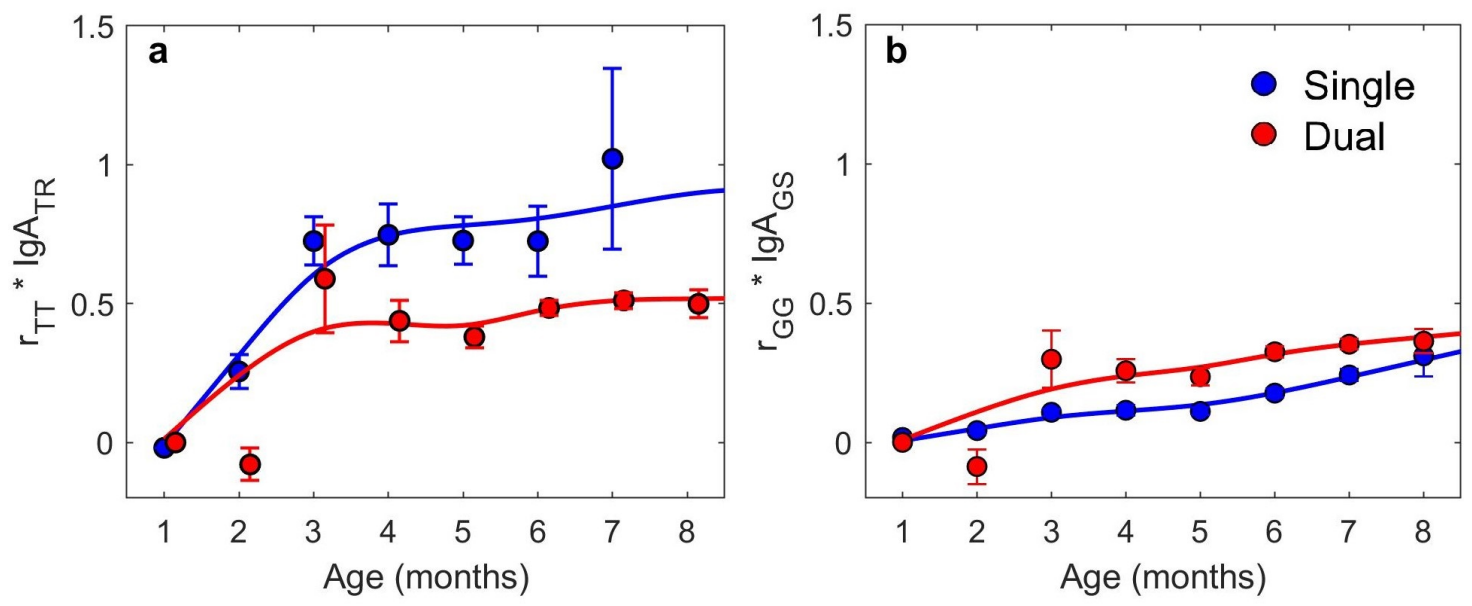

818 Figure 4. Relationship between the contribution of species-specific IgA response, $r_{i i} \operatorname{lgA}$,

819 by host age for T. retortaeformis (a) and G. strigosum (b) in single- (blue) and dual- (red)

820 infected hosts from population $A$ as inferred from the model. The simulated $r_{i i l g A}$ values

821 (mean and S.E.) and the $4^{\text {th }}$ order polynomial curves, weighted by sample size, are 822 reported. Small S.E. are masked by the circles. 

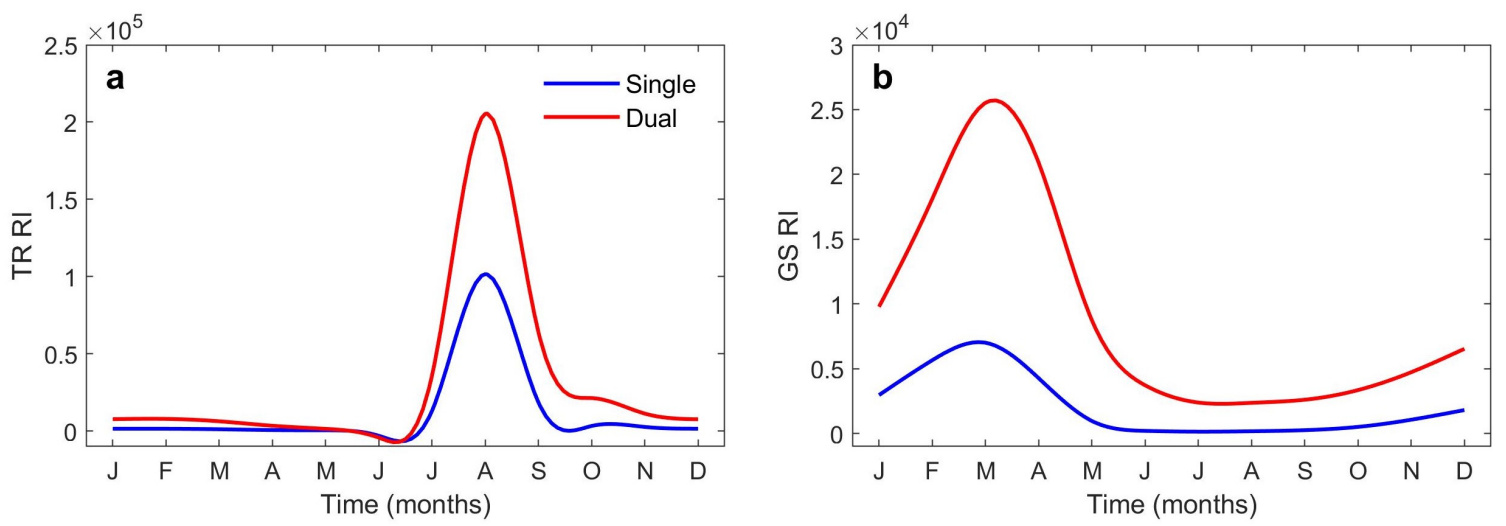

825

826 Figure 5. Estimated seasonality of the mean risk of infection (RI) by sampling month for $T$.

827 retortaeformis (TR, a) and G. strigosum (GS, b) in single- (blue) and dual-infected (red) hosts 828 from population $\mathrm{A}$. 